\title{
LA T DORADA: ESTUDIO EN COMUNIDAD DE UN NUEVO DISPOSITIVO INTRAUTERINO EN COLOMBIA
}

\author{
Dr. Mario Gartner* \\ Dr. Mario Calle** \\ Dr. Alcides Estrada*** \\ Dr. Anthony R. Measham***
}

A pesar de la efectividad y aceptabilidad comprobadas de dispositivos intrauterinos (DIUs) tales como el Lippes, durante mucho tiempo los investigadores han buscado modelos perfeccionados con tasas bajas de embarazo, retiro y expulsión. Uno de los dispositivos llamados de la "segunda generación" es la T-Dorada, cuya distribución ha sido ya aprobada en algunos países europeos. Este informe describe una investigación realizada en Colombia sobre la TCU-200 y que formó parte de un estudio comparativo adelantado en cinco países sobre la efectividad del nuevo dispositivo al utilizarlo bajo las condiciones existentes en la comunidad. El estudio fue realizado por la Universidad de Caldas.

En 1967 el Dr. Howard Tatum desarrolló un DIU de plástico inerte, en forma de $T$, que producía bajas tasas de expulsión y retiro, combinaclas con una eficacia anticonceptiva moderada (1). Por la misma época, en Chile, el Dr. Jaime Zipper y sus colegas demostraron los efectos anticonceptivos del cobre $(2,3)$. Después de realizar pruebas de laboratorio para comprobar la inocuidad del cobre al utilizarlo en seres humanos, se en- rolló un alambre de este material alrededor del brazo vertical de la $T$ para formar el dispositivo Ilamado T-Dorada.

En el caso de la TCu-200, el alambre ofrece una superficie de cobre de $200 \mathrm{~mm}$.

\section{Diseño de la investigación}

A partir de Agosto de 1971 se ofreció el dispositivo TCu-200 a las mujeres que solicitaban DIUs en ocho clínicas situadas en una zona determinada de los Departamentos de Caldas y Risaralda. El grupo de estudio sobre el cual se basa este informe incluyó a las 914 mujeres que habían aceptado el dispositivo hasta Noviembre 1, 1971. En Junio de 1972 se programaron entrevistas con $788 \mathrm{mu}$ jeres del área urbana y suburbana, de las cuales se efectuaron a satisfacción 630 entrevistas ( $80 \%$ ). Además, se consiguieron datos clínicos para otras 237 pacientes, quienes habían visitado la clínica al menos una vez después de la inserción del dispositi-

\footnotetext{
* Investigador principal.

** Coordinador del proyecto.

$* *$ Consultores.
} 
vo, totalizando así información de control para 867 mujeres (95\% del grupo estudiado).

\section{Resultados}

La Tabla 1 muestra la edad y número de hijos vivos de las 914 mujeres en el momento de aceptación y el porcentaje de mujeres entrevistadas en cada grupo de paridad. (Para los efectos de este informe, se define paridad como número de hijos vivos). Sesenta y dos por ciento de las mujeres eran de edad inferior a los 30 años mientras que 52 por ciento tenían tres o menos hijos vivos; $26 \%$ de las mujeres tenían seis o más hijos vivos. En total, se entrevistó a $69 \%$ de las aceptantes, con poca variación en el porcentaje por grupo de paridad.

Ochenta y tres por ciento de las pacientes se clasificaron como habitantes urbanas y $17 \%$ como habitantes rurales. Anteriormente habían practicado la anticoncepción $57 \%$ de las mujeres, de éstas, $20 \%$ habían utilizado un DIU y $40 \%$ anticonceptivos orales. El preguntárseles cuantos hijos deseaban tener, $81 \%$ respondieron que no deseaban más hijos.

\section{TABLA 1}

EDAD DE LAS ACEPTANTES, NUMERO DE HIJOS VIVOS, $Y$ PORCENTAJE ENTREVISTADO EN CADA GRUPO DE PARIDAD

\begin{tabular}{|c|c|c|c|c|}
\hline Edad & Porcentaje & Hijos Vivos & Porcentaje & $\begin{array}{c}\text { Porcentaje entrevis- } \\
\text { tado por grupo } \\
\text { de paridad }\end{array}$ \\
\hline $15-19$ & 9 & 0,1 & 17 & 66 \\
\hline $20-24$ & 25 & 2 & 19 & 70 \\
\hline $25-29$ & 28 & 3 & 16 & 71 \\
\hline $30-34$ & 20 & 4 & 12 & 71 \\
\hline $35-39$ & 13 & 5 & 10 & 70 \\
\hline $40+$ & 5 & $6+$ & 26 & 68 \\
\hline TOTAL & 100 & & 100 & 69 \\
\hline $\mathrm{n}=914$ & & & $\mathrm{n}=914$ & \\
\hline
\end{tabular}

La Tabla 2 muestra los cálculos, según la metodología de la tabla de vida (4), de la tasa acumulada de continuidad de nueve meses y la tasa acumulada de terminación según su causa, basándose en los datos obtenidos en las entrevistas y los registros clínicos. Al considerar únicamente los datos tomados de las entrevistas, se notan apenas leves diferencias en las tasas presentadas en la Tabla 2, en todos los casos, menos del 2 por ciento (5).
La Tabla 3 presenta las tasas acumuladas de continuidad, según la tabla de vida, de acuerdo a la edad y paridad en el momento de aceptación.

Obviamente resulta interesante comparar la efectividad del TCu-200 con la de otros DIUs empleados en Colombia. Los únicos datos para comparación provienen de una encuesta de seguimiento realizada en 1970 entre aceptantes del dispositivo Lip- 
Vol. $X X V$

No 4 LA T DORADA: ESTUDIO DE UN NUEVO DISPOSITIVO INTRAUTERINO EN COLOMBIA

TABLA 2

TASAS ACUMULADAS DE CONTINUIDAD Y TERMINACION PARA EL PRIMER SEGMENTO DE TCU-200

\begin{tabular}{lcc}
\hline & Tasas de Continuidad & \\
& & Porcentaje \\
\hline 6 & & 86.9 \\
9 & 82.7
\end{tabular}

Tasa de terminación de nueve meses según causa

\begin{tabular}{lr} 
Embarazo & 1.8 \\
Expulsión & 3.4 \\
Retiro* & 12.0 \\
Desconocido & 0.1 \\
\hline Total & 17.3 \\
Retiro Médico* & \\
Dolor & 2.6 \\
Sangre & 4.1 \\
Infección & 0.5 \\
Otras causas médicas & 1.8 \\
Retiro no médico* & 2.9 \\
$\mathrm{n}=914$ & \\
\hline
\end{tabular}

TABLA 3

TASAS ACUMULADAS DE CONTINUIDAD DE NUEVE MESES PARA PRIMER SEGMENTO DE TCU200 SEGUN EDAD Y NUMERO DE HIJOS VIVOS EN EL MOMENTO DE ACEPTACION

\begin{tabular}{lccc}
\hline $\begin{array}{c}\text { Edacl en } \\
\text { años }\end{array}$ & $\begin{array}{c}\text { Tasa } \\
\text { (porcentaje) }\end{array}$ & $\begin{array}{c}\text { Número de } \\
\text { hijos vivos }\end{array}$ & $\begin{array}{c}\text { Tasa } \\
\text { (porcentaje) }\end{array}$ \\
\hline $15-19$ & 66.4 & 0.1 & 73.1 \\
$20-24$ & 78.3 & 2 & 79.4 \\
$25-29$ & 79.3 & 3 & 76.8 \\
$30-34$ & 87.0 & 4 & 82.4 \\
$35-39$ & 87.0 & 5 & 84.8 \\
$40-44$ & 91.7 & 6 & 88.8 \\
& & $7+$ & 90.3 \\
$\begin{array}{l}\text { TOTAL } \\
n=630\end{array}$ & 81.9 & & 81.9 \\
\hline
\end{tabular}

pes en varios programas hospitalarios post-parto (5). La muestra consistió en 552 usuarias del Lippes en 11 hospitales, de las cuales se entre- vistó el $54 \%$. Se dispuso de datos clínicos adicionales para mujeres no entrevistadas, lo cual elevó el nivel cubierto al $86 \%$ (475 mujeres). Como para el Lippes se calcularon las tasas de continuidad para todos los segmentos, éstas se compararán con las tasas de dos segmentos correspondientes a la T-Dorada.

En la Tabla 4 se comparan las tasas acumuladas de continuidad para los dos dispositivos según número de hijos vivos en el momento de aceptación.

En la Tabla 5 se comparan el dispositivo Lippes y el TCu-200 según las causas de suspensión (5).

Cuando las tasas de continuidad de 10 meses se estandarizan para ambos dispositivos en 3.5 hijos, el dispositivo Lippes presenta una tasa del 76.1 por ciento en comparación con el 82.4 por ciento de la T-Dorada para las mujeres entrevistadas en cada grupo. Esta diferencia tiene significancia estadística $(p<.05)$, pero es posible que las distorsiones debidas a la no respuesta hayan afectado estos resultados.

\section{Discusión}

La Tabla 5 muestra tasas de continuidad de 10 meses de 83.1 por ciento para la TCu-200 y de 78.7 por ciento para el dispositivo Lippes: la diferencia no reviste significancia estadística, como tampoco la tiene la tasa de embarazo inferior que se presenta en el caso de la TCu-200. No obstante, la tasa inferior de expulsión para la T-Dorada sí es significativa $(p<.01)$ aunque esta diferencia se explica parcialmente por la mayor proporción de inserciones postparto del dispositivo Lippes, ya que en este período se presenta una ma- 
TABLA 4

COMPARACION DE LAS TASAS ACUMULADAS DE CONTINUIDAD DE DIEZ MESES PARA EL DIU LIPPES Y TCU-200 POR NUMERO DE HIJOS VIVOS

\begin{tabular}{ccc}
\hline $\begin{array}{l}\text { Número de Hijos Vivos en } \\
\text { el momento de Aceptación }\end{array}$ & $\begin{array}{c}\text { Tasa de Continuidad }(\%) \\
\text { Lippes } \\
\text { (Todos los seg.) }\end{array}$ & $\begin{array}{c}\text { TCu-200 } \\
(\mathbf{2} \text { seg.) }\end{array}$ \\
\hline 0,1 & 66.1 & 78.4 \\
2 & 73.8 & 82.5 \\
3 & 76.2 & 75.8 \\
4 & 87.3 & 80.8 \\
5 & 67.1 & 84.8 \\
\end{tabular}

TABLA 5

COMPARACION DE TASAS DE SUSPENSION DE DIEZ MESES SEGUN SU CAUSA PARA DISPOSITIVO LIPPES Y TCu-200

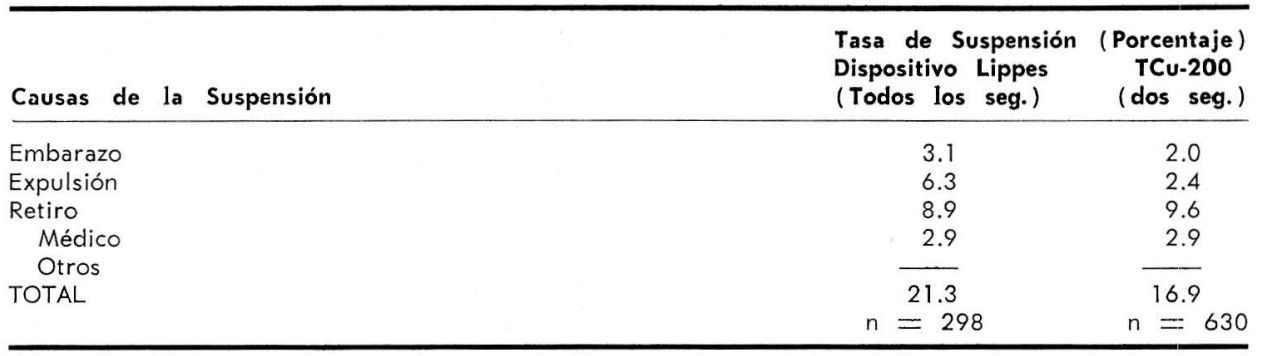

yor probabilidad de expulsión y sólo $30 \%$ de las inserciones de TCu-200 se practicaron en pacientes post-parto.

Para todos los DIUs, la causa más importante de suspensión es el retiro, y ninguno de los dos dispositivos demostró ventaja en este sentido. Cabe anotar que los dos grupos de estudio no son absolutamente comparables, ya que las aceptantes del Lippes se tomaron de programas hospitalarios post-parto, mientras que $70 \%$ de las pacientes con T-Dorada provenían de la población en general. Era de esperar que la amenorrea postparto redujera el número de embarazos con el dispositivo Lippes, pero las cifras correspondientes a la T-Dorada no muestran diferencias importantes en las tasas de embarazo para mujeres que recibieron el dispositivo antes 0 después de reanudada la menstruación (6). Los retiros por hemorragia y dolor son más frecuentes, al menos inicialmente, en los casos de inserciones no practicadas después del parto, de tal forma que en este respecto la comparación de los dos grupos puede resultar injusta para la T-Dorada. Ciertamente, los médicos participantes en el estudio opinaron que la T-Dorada es insertada con mayor facilidad, y su impresión clínica era que producía menos efectos secundarios que el dispositivo Lippes. 
Las tasas de continuidad aparentemente similares presentan diferencias interesantes al considerarlas según el número de hijos vivos en el momento de aceptación. (Véase Tabla 4 ). Se observa que las tasas correspondientes a la T-Dorada son más altas para las mujeres en los grupos de 0-2 y $5 \circ$ más hijos, mientras que el dispositivo Lippes presenta la tasa más alta para las mujeres con 4 hijos. La T-Dorada se destaca en 4 de los seis grupos de paridad que se comparan, y el dispositivo Lippes en uno, siendo igual la tasa en el restante grupo para los 2 métodos.

La Tabla 4 muestra que las aceptantes de la T-Dorada presentan tasas de continuidad más altas según edad y paridad creciente, como era de esperar, pero los datos contenidos en la misma tabla demuestran, no obstante, una ventaja considerable sobre el disposistivo Lippes en tasas de continuidad para mujeres con menor número de hijos (0-2).

\section{Conclusiones}

Este estudio en comunidad de la T-Dorada indica cierta ventaja de esta sobre el dispositivo Lippes, en lo que se refiere a tasas de continuidad y expulsión. La diferencia en las tasas de embarazo no tienen significancia estadística. Cuando las tasas de continuidad se estandarizan según paridad de 3.5, la TCu-200 parece tener tasas más altas en el nivel de 10 meses, que el dispositivo Lippes (82.4 versus 76.1 por ciento) (5). No obstante, los datos para los dos dispositivos no son estrictamente comparables. Primero, entre las aceptantes del dispositivo Lippes hubo un nivel más alto de no-respuesta, y aproximadamente el doble de las pacientes estaban en el período postparto. Además, el estudio sobre la TCu-200 se llevó a cabo dos años después del estudio correspondiente al dispositivo Lippes utilizando una muestra regional, y no nacional como en el caso del Lippes. Estos factores podrían restar importancia a las diferencias encontradas en el presente estudio. No obstante, la T-Dorada demostró mayor aceptabilidad que el dispositivo Lippes en los casos de mujeres con menos de tres hijos, según se observa en las tasas de continuidad.

Actualmente se recomienda que la TCu-200 sea reemplazada después de tres años in útero, pues aun se desconoce la verdadera vida efectiva del cobre. Aún dentro de esta limitación, la T-Dorada parece ofrecer ventajas considerables para las mujeres de baja paridad que deseen espaciar sus familias. Actualmente se están realizando investigaciones sobre nuevos modelos de T-Dorada que se espera tendrán una vida más larga. Si se logra ese objetivo, la T-Dorada constituirá una valiosa contribución a los métodos anticonceptivos existentes.

\section{Conclusions}

This community trial of the Cooper$T$ IUD indicates some advantage of the device over the Lippes loop in terms of continuation and expulsion rates. The difference in pregnancy rates is not statistically significant. When continuation rates are standardized by parity to a median of 3.5 , the TCu-200 appears to have significantly higher 10 month rates than the Lippes loop (82.4 versus 76.1 percent). However, the data for the two devices are not strictly comparable. First, there was greater non-response among loop acceptors and aproximately twice as many accepted postpartum. Furthermore, the TCu-200 study was conducted 2 years later using a regional instead of a national sample as in the case of the loop. These factors may make the differences found 
Julio-Agosto 1974

Rev. Col. Obst. y Ginec.

in this study less important than they appear. Nonetheless, the Cooper-T proved to be more acceptable than the Lippes loop to women with fewer than three children, as judged from the continuation rates.

At the present time it is recommende dthat the TCu-200 be rplaced after 3 years in utero, since the effective life or the copper is not known with certainty. Even with this limitation, the device would seem to offer distinct advantages to low parity women seeking to space their families. Research is currently being carried out on models expected to have a much longer life. If this objective is archieved, the Copper-T should represent a useful addition to the contraceptive methods now available.

\section{AGRADECIMIENTOS}

Los autores quieren expresar su sincero agradecimiento a las muchas personas de la Universidad de Caldas, la Asociación Colombiana de Facultades de Medicina, y el Consejo de
Población que colaboraron en el trabajo de campo y en el procesamiento de datos para cste proyecto.

Las separatas deben ser solicitadas al $\mathrm{Dr}$. Alcides Estrada en la siguiente dirección:

Dr. Alcides Estrada

Contro Regional de Población

Apartado Aéreo 24846

Bogotá

\section{BIBLIOGRAFIA}

1 TATUM, H. J.: Am. J. Obstet. Gynecol. 112: 1004, 1972.

2 ZIPPER, J. A., MEDEL, M., y PRAGER, R. : Abstracts: Sixth World Congress on Fertility and Steril. (Abstractos: Sexto Congreso Mundial sobre Fecundidad y Esterilidad). Tel Aviv, Israel, Mayo 1968. p. 154.

3 ZIPPER, J. A., MEDEL, M., y PRAGER, R. : Am. J. Obstet. Gynecol. 105: 529, 1969.

4 FREEDMAN, R., y TAKESHITA, Family Plan-ning in Taiwan: An Experiment in Social Change (La Planificación Familiar en Taiwan: Un Experimento para el Cambio Social). (Princeton: Princeton University Press, 1969).

5 SIVIN, I. Studies in Family Planning, 4, No 7: 162-170, 1973.

6 SIVIN, I. datos inéditos. 